\title{
PARA UNA DELIMITACIÓN DEL EFECTO EXPANSIVO DE LOS CONTRATOS. COMENTARIO A LA SENTENCIA DE LA CORTE SUPREMA DE 25 DE ENERO DE 2011 CASACIÓN EN EL FONDO ROL N³738-2009
}

\author{
THE LIMITS OF THE EFFECTS OF CONTRACTS ON THIRD PARTIES. \\ A COMMENT REGARDING CHILEAN SUPREME COURT JUDGMENT'S \\ ON JANUARY $25^{\text {TH }} 2011$
}

\section{Ian Henríquez Herrera*}

\begin{abstract}
RESUMEN: En este comentario nos proponemos mostrar cómo los contratos producen efectos respecto de terceros, y cómo tales efectos requieren una delimitación. Con tal objeto, identificamos, describimos y analizamos diferentes casos en que los contratos producen efectos a terceros no contratantes. A partir de ello, proponemos una nueva sistematización del así llamado "efecto absoluto" de los contratos, en la que destaca el efecto expansivo. Finalmente, y en el mismo sentido, analizamos una reciente sentencia de la Corte Suprema referida a la misma materia.
\end{abstract}

Palabras clave: Daños contractuales a terceros, efecto absoluto del contrato, efecto expansivo.

ABSTRACT: In this article, we expect to show how contracts have effects regarding third parties and how such effects require some delimitation. In doing so, we identify, describe and analyze several cases in which agreements produce effects to no parties. Therefore, we propose a new system of concepts to explain the effects of contracts, specially the named "absolute effect". In this system, the efecto expansivo has a preeminent place. Finally, we analyze a recent judgment from the Chilean Supreme Court regarding this topic.

Key words: Contractual damages and losses to third parties, privity of contract exceptions, contracts effects to third parties.

\section{INTRODUCCIÓN}

En el presente trabajo pretendemos delimitar el efecto expansivo del contrato. Para ello, habremos de intentar una conceptualización y sistematización del así llamado efecto absoluto. Con tal objeto, en primer término describiremos los aportes de la doctrina nacional sobre el particular, observando aquellos aspectos que, pensamos, son susceptibles de un mayor depuramiento. Sostendremos el efecto absoluto como un efecto típicamente contractual, que se distingue de las excepciones al efecto relativo y que admite diferentes especificaciones. Entre estas, mostraremos el deber de reconocimiento del contrato ajeno, el efecto erga omnes, el efecto reflejo, y el efecto expansivo. Este último es el que concentrará

* Profesor de la Universidad Central y de la Universidad de los Andes.

Licenciado en Derecho y Magíster en Derecho Privado, Universidad de Chile; Magíster en Investigación Jurídica y Doctor en Derecho, Universidad de los Andes; Abogado. 
especialmente nuestra atención y análisis, a partir de un fallo de la Corte Suprema que casó una sentencia por vicios de fondo sobre la base del extender las reglas de responsabilidad contractual a un tercero relacionado. Nuestra tesis es que el efecto expansivo puede cumplir un rol especial en la protección de las víctimas de daños derivados de incumplimiento contractual distintas de las partes.

\section{PLANTEAMIENTO DEL PROBLEMA}

En nuestra literatura ha sido doctrina común el señalamiento del efecto relativo como uno de los principios de la contratación, expresado en la máxima res inter alios acta ${ }^{1}$ y contenido, especialmente, en el artículo 1545 del Código Civil (CC). Dicha doctrina se remontaría a Domat, cuyo influjo pasó al Code, en el que hay texto expreso a este respecto (artículo 1165) ${ }^{2}$. En Francia, a partir de un agudo estudio de Savatier ${ }^{3}$, dicha doctrina quedó en entredicho, y tal crítica llegó hasta nosotros por intermedio de Ramón Domínguez Águila ${ }^{4}$. Luego, Jorge López Santa María, en su tratado sobre los contratos, argumenta en pro del efecto absoluto, que denomina indistintamente reflejo o expansivo, y le trata en conjunto con la oponibilidad ${ }^{5}$. En tiempo más reciente, Carlos Pizarro ha identificado una serie de situaciones que corresponderían a este tipo de efecto de los contratos ${ }^{6}$. Estos tres autores tienen en común haber perfeccionado su formación bajo la órbita francesa. Álvaro Vidal, adscrito en cambio al influjo español -lugar en el que también hay norma expresa (artículo 1257 Código Civil español $^{7}$ )-, ha hecho un esfuerzo de sistematización ${ }^{8}$, que pudiera parecer excesivo, como ya ha sido sugerido por otro doctrinario?.

En primer término, hemos de constatar que el CC no contiene norma alguna que expresamente aluda al efecto relativo del contrato -como sí la contienen los códigos civiles español y francés-. De ello se siguen dos consecuencias estrechamente relacionadas. La primera, que si en aquellos sistemas en los cuales hay norma expresa que restringe el efecto de los contratos a las partes, ha habido desarrollo del concepto dogmático de efecto absoluto de los contratos, con mayor razón ello debiese ser así en nuestro sistema -dado que no tenemos restricción de texto-. La segunda, que aquellas sistematizaciones que se han hecho a partir precisamente de la restricción de texto -como por ejemplo, la diferencia entre efectos

${ }^{1}$ Cod. Just. 7.60.1; 7.56.4; 7.60.2: res inter alios acta non posse facere praeiudicium.

2 Artículo 1165 C.C. francés: Los pactos no tienen efecto alguno, sino entre las partes contratantes: no pueden perjudicar ni aprovechar a un tercero, sino en el caso prevenido en el artículo 1121 [estipulación a favor de un tercero].

3 Savatier (1934) pp. 525 ss.

4 Domínguez (1983) pp. 151 a 165.

5 López Santa María (2005) t.2, p. 364.

6 Pizarro (2007) pp. 551 a 567.

7 Art. 1.257 C.C. español: Los contratos solo producen efecto entre las partes que los otorgan y sus herederos; salvo, en cuanto a estos, el caso de que los derechos y obligaciones que proceden del contrato no sean transmisibles, o por su naturaleza, o por pacto, o por disposición de la ley. / Si el contrato contuviere alguna estipulación en favor de un tercero, este podrá exigir su cumplimiento, siempre que hubiese hecho saber su aceptación al obligado antes de que haya sido aquella revocada.

8 Vidal (2006) pp. 51 a 85.

9 Pizarro (2007) pp. 555-556. 
directos e indirectos del contrato, propia de la doctrina española- pueden resultar excesivas para nuestro sistema.

\section{EL EFECTO ABSOLUTO COMO UN EFECTO NORMAL DE LOS CONTRATOS}

Los autores nacionales ya citados -Domínguez A., López Santa María, Carlos Pizarro y Álvaro Vidal- han mostrado de manera suficiente que no es correcto sostener que los contratos solo producen efecto entre las partes. Por el contrario, como expresamente lo indica Vidal, la oponibilidad del contrato a terceros es la regla general. El argumento, en este punto, es simple y es de tipo a contrario sensu: si el CC contempla normas expresas sobre situaciones a las que sanciona con inoponibilidad, es en el entendido que la regla general es la contraria ${ }^{10}$. Es un argumento utilizado en España por Díez-Picazo y otros, que resulta de toda lógica y que ha sido construido sobre normas análogas a las chilenas, de modo tal que, nos parece, también es válido para nosotros. Por ende, cabe sostener que los contratos producen efectos no solo respecto de las partes, sino también respecto de terceros. Esto es lo que, en términos generales, denominamos efecto absoluto. Por ende, sería admisible la sinonimia -en este contexto y para estos fines- entre efecto absoluto y oponibilidad ${ }^{11}$.

De lo anterior se sigue que, en rigor, el efecto absoluto no es una excepción al efecto relativo, sino que un efecto distinto derivado del mismo contrato. Por otra parte, esto nos permite excluir del efecto absoluto aquellos efectos derivados de las anomalías o patologías contractuales -como la acción de simulación o de nulidad del contrato ejercida por terceros-.

\subsection{El EFECTO ABSOLUTO NO ES UNA EXCEPCIÓN AL EFECTO RELATIVO}

Para evitar confusiones en este punto, insistiremos sobre una cuestión de Perogrullo: dado que el efecto absoluto no es una excepción al efecto relativo, de ello se sigue que las excepciones al efecto relativo no pueden conceptuarse como manifestaciones del efecto absoluto. Entre estas excepciones, siguiendo a López Santa María, situamos la estipulación a favor de otro y la promesa de hecho ajeno ${ }^{12}$. Estas figuras negociales quedan, entonces, fuera del ámbito de nuestro análisis circunscrito al efecto absoluto.

Ahora bien, junto con lo anterior, deben excluirse del efecto absoluto las acciones de contratos anómalos, según pasamos a explicar.

\section{2. El efecto absoluto difiere de las acciones de contratos anómalos}

La afirmación referida a que el efecto absoluto es un efecto normal de los contratos es razonable y justificada. Si ello es así, debe lógicamente excluirse como manifestación

\footnotetext{
${ }^{10}$ Diversas disposiciones del CC aluden a los efectos respecto de terceros: 1882 (pacto de retrocompra), 1723 (convención matrimonial), etc.

11 Con esto, nos distanciamos de la doctrina española, que distingue primero entre efectos directos -entre las partes- e indirectos - para con terceros-. Entre estos últimos, a su vez, distingue entre efectos reflejos y aquellos provocados frente a terceros, y entre estos, la oponibilidad y la utilizabilidad del contrato. Cfr. Díez-Picazo (1996) pp. 427-428.

${ }^{12}$ López Santa María (2005) pp. 350 y 361.
} 
del efecto absoluto la legitimación activa de terceros de acciones que impugnan la validez de un contrato, tales como la acción de simulación ${ }^{13}$ o la acción de nulidad ${ }^{14}$. En ambos casos, por definición, nos encontramos ante situaciones normativamente irregulares, con prescindencia de su prevalencia práctica.

De lo dicho, se desprende que podemos identificar el efecto relativo, excepciones al mismo -estipulación a favor de otro, promesa de hecho ajeno- y patologías contractuales -nulidad, simulación-; además del efecto propiamente absoluto. En lo que sigue, intentaremos una sistematización de este último.

\section{UN INTENTO DE SISTEMATIZACIÓN DEL EFECTO ABSOLUTO}

Ahora bien, nos parece que en nuestra literatura coexisten dos visiones a la hora de tratar sobre el efecto absoluto. Una, hasta donde atisbamos de influjo francés, que prefiere un tratamiento global e indiferenciado de los efectos contractuales sobre terceros (en la que podemos situar a Ramón Domínguez A., Jorge López y Carlos Pizarro), y otra, de base española, que presenta una serie de disquisiciones que no nos resultan del todo ni convincentes ni clarificadoras (en la que podemos situar a Álvaro Vidal). Sobre la base de los importantes aportes de estos autores, intentaremos una delimitación que, esperamos, se ajuste del mejor modo posible al derecho positivo nacional vigente.

Dado que nuestro oficio es un arte de la distinción y es precisamente el lenguaje el que nos permite dar cuenta de las diferencias, el uso indistinto de términos tales como efecto absoluto, efecto reflejo o efecto expansivo -que podemos advertir, por ejemplo, en López Santa María- puede ser objeto de una mayor especificación, reservando cada término para situaciones diversas. En este sentido, la adaptación a nuestro sistema de la doctrina de órbita francesa -solo en ahorro de lenguaje y de manera funcional- es susceptible de más distinciones.

Por otra parte, la doctrina de órbita española -se sobreentiende, asimismo, que es una expresión de mera utilidad a los fines de la exposición- presenta distinciones que parecen excesivas y por ende disfuncionales a nuestro sistema. Tal es el caso, nos parece, de efectos directos y efectos indirectos del contrato. El efecto absoluto es rigurosamente un efecto directo hacia terceros.

\subsection{El deber de Reconocimiento del CONTRATO AJENo: El CONTRATO COMO Un HECHO JURÍDICO \\ Una primera manifestación del efecto absoluto deriva del reconocimiento de la reali-} dad del contrato en tanto hecho social, punto que ha sido tratado por todos los autores ya aludidos. De ello se deriva un efecto propiamente jurídico: el deber de reconocimiento de su existencia, sea a favor del tercero, como, en su caso, en contra del tercero -con el límite de la proscripción del perjuicio de derechos con causa de adquisición anterior al contrato ajeno- ${ }^{15}$.

\footnotetext{
${ }^{13}$ En contra, Vidal (2006) p. 83.

${ }^{14}$ Sobre la legitimación activa de terceros en el ejercicio de esta acción véase: CorRAL (2010) p. 115.

15 VIDAL (2006) p. 66.
} 
El incumplimiento contractual, asimismo, puede conceptuarse como un hecho -en tanto que tiene existencia susceptible de ser verificada-, de modo tal que es, asimismo, susceptible de ser invocado por un tercero como causa de un daño en sede extracontractual. Es el caso típico de víctimas por repercusión de un daño acaecido por incumplimiento, por ejemplo, de un contrato de transporte o de prestación de servicios médicos.

Por otra parte, el contrato ajeno es base de interpretación contractual -dado que las cláusulas usuales se presumen, ex artículo 1563 inc. $2^{\circ}-$ e integra además los usos y costumbres propios de la buena fe (ex artículo 1546).

\subsection{El efecto ERGA omNES: El contrato con efectos reales y el Contrato QUE CONFIERE ESTATUS}

Una segunda manifestación del efecto absoluto viene dada por el contrato con efectos reales y por el contrato que confiere estatus. Respecto del primero, si lo propio del derecho real es su oponibilidad erga omnes -según doctrina común-, y el efecto propio del contrato es el nacimiento de derechos y obligaciones, un contrato que genere derechos reales necesariamente ha de tener efectos erga omnes. Daniel Peñailillo ya ha mostrado de manera suficiente cómo en Chile el contrato puede tener efectos reales ${ }^{16}$. Por lo pronto, el contrato con cláusula de tradición ficta (ex artículo $684 \mathrm{~N}^{\circ} 4 \mathrm{CC}$ ) y las servidumbres voluntarias (ex artículo 698 CC) son dos ejemplos de lo anterior.

Respecto del segundo tipo de contratos -aquellos que confieren estatus-, también por definición sus efectos son oponibles a terceros. Nos parece que este punto se visualiza todavía de mejor forma si se atiende a la noción clásica de estatus, que por lo demás es la que pasó a nuestra codificación (ex artículo 304); es decir, la posición jurídica que ocupa una persona -no circunscrita ni constreñida a las relaciones de familia-. Con todo, aun en una aproximación restrictiva del estado civil, el efecto absoluto se manifiesta de todas formas.

\subsection{El EFECTO REFlejo: Los CONTRATOS SUbORDinados, LOS SUbCONTRATOS y LOS CONTRATOS COLIGADOS \\ Como una tercera manifestación específica del efecto absoluto, podemos identificar} el efecto reflejo, es decir aquellos casos en que un contrato proyecta sus efectos a otros pactos que forman parte de una relación obligacional conexa. Entre tales, podemos distinguir: los contratos subordinados, los subcontratos y los contratos coligados.

\subsubsection{Los contratos subordinados}

Los contratos subordinados o dependientes implican una relación jurídica en virtud de la cual el decaimiento de un contrato incide de manera directa en la ineficacia de otro, aun cuando las respectivas partes sean personas distintas. Los contratos de garantía son una buena muestra de esta situación. Estos casos son de fácil comprensión, en virtud de que lo accesorio sigue la suerte de lo principal. Decaído el principal, decae, entonces, el subordinado.

16 Peñailillo (1979) pp. 49-60. 


\subsubsection{Los subcontratos}

A partir de una relación contractual específica puede derivarse otra en la que coincida el objeto del contrato y en la que el deudor original pase a ocupar el rol de acreedor, o viceversa. Es el caso de la subcontratación. En materia civil, figuras usuales en este punto son el subcontrato de arrendamiento (ex artículo 1946) y la delegación del mandato (ex artículo 2135). El contratante principal y el subcontratista no tienen vínculo contractual alguno entre ellos. Sin embargo, la ineficacia del contrato principal produce efectos en el subcontrato, dado que decae la causa de este último ${ }^{17}$.

\subsubsection{Los contratos coligados}

Este, probablemente, sea el caso más interesante y menos estudiado de efectos reflejo de los contratos. Los contratos coligados o cadenas contractuales implican una secuencia de relaciones contractuales en las que una parte ocupa, funcionalmente, un rol de gozne para uno y otro contrato, entre los cuales puede haber identidad de naturaleza jurídica o clara complementariedad económica. La facti specie es múltiple en las sociedades contemporáneas. No obstante ello, casos típicos son las compraventas o suministros sucesivos. En derecho comparado, y también en la doctrina nacional, la tendencia es a conceder acción contractual a quienes estén en los vértices de la cadena ${ }^{18}$.

\subsection{El efecto expansivo: El contrato con EFecto protector a tercero RELACIONADO}

Llegado este punto, nos parece que todavía podemos delimitar de manera justificada un efecto contractual hacia terceros distinto de los casos previamente indicados. Es el efecto que denominamos expansivo y que correspondería al contrato con efecto protector a tercero relacionado, propio de la doctrina alemana ${ }^{19}$. En virtud de este efecto, un tercero puede ejercer acción contractual directa en contra de quien no ha sido su contraparte, pero cuyo incumplimiento le ha afectado en razón de una relación jurídica preexistente y de naturaleza diversa a la contractual. Así, por ejemplo, quien vive junto a un arrendatario y padece los perjuicios del incumplimiento contractual del arrendador, tendría acción contractual directa por los perjuicios. Por cierto, la admisión de este efecto expansivo requiere una rigurosa delimitación. Siguiendo a Medicus, a propósito del efecto protector para tercero, pueden ser criterios para esta tarea: (i) la misma intensidad de exposición al riesgo entre el acreedor y el tercero; (ii) interés especial del acreedor en la protección del tercero; (iii) conocimiento de (i) y (ii) por parte del deudor ${ }^{20}$. Separándonos, ahora, del autor citado, incluimos como criterio de delimitación la concurrencia de los requisitos de la responsabili-

17 No nos interesa en este punto el régimen de subcontratación en la construcción ni el régimen de subcontratación laboral, puesto que es estos casos el efecto absoluto del contrato no parece derivar de la estructura misma de las relaciones obligacionales, sino del régimen legal especial. Dicho de otro modo, derogadas las normas especiales, decaería el efecto absoluto.

${ }^{18}$ Larroumet (i998) p. 32; Pizarro (2007) p. 566; López Santa María (2005) p. 369.

${ }^{19}$ Medicus (1995) pp. 356 a 359.

${ }^{20}$ Medicus (1995) pp. 358-359. 
dad contractual, de modo tal que no bastaría el daño a causa del incumplimiento, sino que se ha de requerir, asimismo, el factor de imputación.

El efecto expansivo tiene justificación dogmática en tanto subsista la diferencia en los regímenes contractuales de responsabilidad contractual y extracontractual respecto de la presunción de culpa. No puede desatenderse que la tendencia jurisprudencial a objetivar la responsabilidad extracontractual en algunos sectores (como por ejemplo, daños ocasionados por dependientes, o accidentes del trabajo) torna superflua la aceptación del efecto expansivo. En efecto, el tercero afectado por el incumplimiento contractual tendrá de todas formas acción aquiliana contra el deudor incumplidor. El punto está, nos parece, en que la tendencia a objetivar por vía jurisprudencial la responsabilidad extracontractual es errada, y debe tornarse al cauce de la imputación subjetiva. Y en tal contexto sí que sería funcional el efecto expansivo.

\section{ANÁLISIS CRÍTICO DE UN CASO JURISPRUDENCIAL}

Con fecha 25 de enero de 2011, la Corte Suprema casó una sentencia de la Corte de Apelaciones de Arica, por errónea aplicación del artículo 1698 del Código Civil ${ }^{21}$. Con ocasión de ello, la Corte Suprema discurre en torno al efecto absoluto de los contratos, a propósito del contrato de cuenta corriente bancaria.

\subsection{Los Hechos}

El núcleo de los hechos es el siguiente: la sostenedora de un colegio particular subvencionado pagó, con un cheque personal suyo, las cotizaciones previsionales de los trabajadores del colegio. El banco librado protestó el cheque, por disconformidad de firma, pese a que el librador era efectivamente quien había suscrito el título de crédito. El atraso en el pago de las cotizaciones implicó multas, reajustes e intereses en contra del colegio, demora en la recepción de subsidios estatales, e inconvenientes ante el Ministerio de Educación. La sostenedora - persona natural que giró el cheque- y el colegio, demandaron conjuntamente al banco, bajo el estatuto de la responsabilidad contractual. En primera y segunda instancia la demanda fue rechazada.

\subsection{Aplicación del efecto absoluto (Expansivo)}

La legitimación activa del colegio estaba en entredicho, al no ser parte en el contrato de cuenta corriente cuyo incumplimiento se alegaba. Por otra parte, el daño de la sostenedora era más bien indirecto o reflejo en relación al colegio. Para ambos problemas, el efecto absoluto de los contratos tenía importante incidencia.

Los considerandos $6^{\circ}$ a $10^{\circ}$ de la sentencia de reemplazo tocan el punto. En primer término, el fallo conceptúa el efecto absoluto, expansivo, indirecto o reflejo:

"SEXTO: Que en efecto, al margen de la voluntad de las partes contratantes, en determinadas circunstancias un contrato puede ser invocado por un tercero en su favor

${ }^{21} \mathrm{~N}^{\circ}$ LegalPublishing: 47547. 
$\mathrm{u}$ opuesto a un tercero en su detrimento. Teniendo presente que un contrato en ciertas circunstancias puede ser un hecho "que como tal existe para todos, en sus efectos reflejos puede alcanzar a terceros absolutos: no para crear directamente un derecho o una obligación en el patrimonio de ellos, sino en cuanto es factible traerlo a colación, o formular una pretensión basada en el contrato ajeno. Esto es lo que se llama el efecto absoluto de los contratos, o efecto expansivo o efecto indirecto o efecto reflejo de los contratos (Álvaro Vidal Olivares, "El Efecto Absoluto de los Contratos en Revista Chilena de Derecho Privado, 6, 2006, pp. 51 y ss. citado por Jorge López Santa María "Los Contratos 5a . Edición, Editorial LegalPublishing)".

Luego, el fallo de reemplazo sostiene una tesis que, aunque de base nos parece correcta, requiere una mayor delimitación: que un contrato es capaz de crear derechos o imponer una obligación a un penitus extranei sin su consentimiento:

“SÉPTIMO: Que siguiendo el raciocinio que expone López Santa María, al referirse a las excepciones al principio del efecto relativo, podemos concluir que un contrato es capaz de crear derechos o imponer una obligación a un penitus extranei sin su consentimiento. De tal forma que el efecto expansivo o efecto absoluto de los contratos no surge de esto un derecho u obligación directamente para el tercero, sino que este puede invocar un contrato ajeno o vérselo o poner en cuanto ese contrato indirectamente lo afecta en su situación jurídica y patrimonial. El efecto expansivo del contrato se sitúa en una perspectiva más amplia que la del principio tradicional del efecto relativo. El efecto expansivo implica una superación de la rigidez y estrechez del efecto relativo, una suerte de abandono o sustitución de los marcos que encuadran la institución del efecto relativo, para situar el análisis jurídico a otro altura, menos dogmática y más realista. (ob. cit. "Los Contratos, pág. 308)".

Con todo, esto muestra ya un notable avance en la difusión y comprensión del efecto absoluto del contrato. Por otra parte, la Corte explicita la vigencia erga omnes del contrato y su realidad en tanto hecho social:

OCTAVO: Que la premisa de la que hay que partir para referirse al efecto expansivo de los contratos es la situación de hecho que sucede en la realidad. Un acontecimiento jurídico del mundo exterior, que nadie puede desconocer y que, por lo tanto, tiene vigencia erga omnes. Así percibida la realidad de las cosas, es posible traer al primer plano de una controversia, como antecedente esencial o fundamental un contrato ajeno. Sin que la correspondiente alegación pueda desestimarse sobre la base del principio del efecto relativo.

NOVENO: Que al respecto el Profesor Domínguez ha dicho en el mismo sentido, que "no es efectivo que el contrato sea inoponible a terceros. Muy por el contrario, el contrato es, por esencia, oponible a todos, porque nadie puede desconocer el contrato convenido entre otros, ni puede, por lo mismo, impedir, ni privar, ni discutir a las 
partes los derechos y obligaciones que provienen del contrato. Lo que ocurre es que el contrato no es únicamente una cuestión de creación de norma legal y de imperio de la voluntad. Se inscribe en un contexto social y, por lo mismo, es también un hecho social... Afirmar, pues, que los contratos son inoponibles a terceros sería una afirmación inexacta que no ayuda a la claridad de las ideas, por mucho que se repita en alguna jurisprudencia... Hay todo un radio de repercusión del contrato que va desde las partes hasta terceros más próximos y otros cada vez más lejanos. Como se ha podido describir, ese efecto es semejante al de la caída de una piedra en el agua, que va formando, desde el centro, anillos cada vez más grandes que terminan, en los mayores y más lejanos, por mover apenas las aguas. Frente a terceros, el contrato tiene una eficacia menor o disminuida; pero la tiene y variada, aunque no para todos esa eficacia sea igual. (Los Terceros y el Contrato, en Revista de Derecho, Universidad de Concepción, 174, 1983, pp.151 y ss.)”.

La Corte, además, sostiene que el efecto absoluto es una noción de cerramiento en el derecho de daños y una institución de carácter subsidiario, análoga al enriquecimiento injusto:

"DÉCIMO: Que "el efecto reflejo de los contratos es una noción de cerramiento en el Derecho de daños, que permite solucionar problemas huérfanos de regulación legal concreta. Tal como ocurre con el enriquecimiento sin causa, en cuanto la actio in rem verso permite encontrar la fuente de una obligación determinada, que no ha nacido ni de un contrato, cuasicontrato, delito, cuasidelito ni de la ley, o sea de ninguna de las fuentes tradicionales a las que se refiere el artículo 1437 del Código Civil. En este sentido, ambas figuras jurídicas, el efecto reflejo de los contratos y el enriquecimiento sin excusa, son instituciones de carácter subsidiario. (ob. cit. López Santa María, pp.153-154)”.

Este último aspecto es el que nos parece de especial relevancia, puesto que, siguiendo de cerca a López Santa María, el fallo reconoce el efecto reflejo -aunque nos parece que se refiere a lo que nosotros denominamos "expansivo"- como una herramienta de subsidio en el derecho de daños - "noción de cerramiento", dice el considerando-. De tal manera que, atendidas las particularidades del caso, es posible sostener la viabilidad del efecto expansivo en el derecho de la contratación chilena.

Ahora bien, la aceptación del efecto expansivo debe cuidarse de desnaturalizar el vínculo obligacional derivado del contrato; sea porque llegue a ignorar el efecto relativo, sea porque termine por concebir al contrato como un mero distribuidor de riesgos económicos. Estos son vértices indeseables. Es posible, en todo caso aunque no sin dificultad, precisar los límites del efecto expansivo, de modo tal de darle la debida aplicación que permita soluciones más justas en casos de incumplimientos contractuales que dañen a terceros relacionados. 


\section{CONCLUSIONES}

El CC chileno no tiene una norma expresa que restrinja los efectos del contrato a las partes, a diferencia de los códigos civiles francés y español. Por consiguiente, la doctrina francesa y la española requieren ser objeto de análisis crítico antes de ser aplicadas entre nosotros.

Por lo pronto, si en Francia y España se ha desarrollado el efecto absoluto de los contratos, con mayor razón habría de admitirse en Chile, dado que no tenemos limitación de texto sobre este punto.

En otro sentido, la sistematización francesa es susceptible de una mayor diferenciación a la hora de ser aplicada en nuestro medio. La española, en cambio, hace disquisiciones que pudieran resultar excesivas.

A partir de esas experiencias, hemos propuesto una sistematización que implica: (i) reconocer el efecto absoluto como un efecto típicamente contractual, que equiparamos a oponibilidad; (ii) diferenciar las excepciones al efecto relativo del efecto absoluto; (iii) distinguir en el efecto absoluto los siguientes tipos: el deber de reconocimiento, el efecto erga omnes, el efecto reflejo, el efecto expansivo.

Finalmente, sostenemos que el efecto expansivo puede operar como un factor de atribución de responsabilidad contractual.

\section{BIBLIOGRAFÍA CITADA}

Corral, Hernán (2010): Contratos y daños por incumplimiento (AbeledoPerrot/LegalPublishing, Santiago)

Díez-Picazo, Luis (1996): Fundamentos de derecho civil patrimonial (Madrid, Civitas) v.1.

Domínguez A., Ramón (1983): "Los terceros y el contrato", en Revista de Derecho (Universidad de Concepción), 174, LI, jul.-dic., pp. 151 a 165.

López Santa María, Jorge (2005): Los contratos (Editorial Jurídica de Chile, Santiago), t. 2 .

Medicus, Dieter (1995) Tratado de las relaciones obligacionales (Bosch, Barcelona) v. 1 , pp. 356 a 359.

Peñailillo, Daniel (1979): "Dificultades del efecto personal del contrato”, en Revista de Derecho (Universidad de Concepción), 167, XLVI, enero-dic., pp. 49-60.

Pizarro, Carlos (2007): "El efecto relativo de los contratos: partes y terceros", en Guzmán, A. (editor): El Código Civil de Chile (1855-2005) (LegalPublishing, Santiago) pp. 551 a 567.

Larroumet, C. (1998): Responsabilidad civil contractual. Algunos temas modernos (Editorial Jurídica de Chile, Santiago).

Savatier, R. (1934): "Le prétendu principe de l'effet relatif des contrats" en Rev. Trim. de Droit Civil, pp. 525 ss.

VIdal, A. (2006): "El efecto absoluto de los contratos", en Revista Chilena de Derecho Privado, 6, pp. 51 a 85. 